\title{
Universidades transparentes con bibliotecas transparentes
}

\author{
Ana R. Pacios *
}

Artículo recibido:

28 de agosto de 2014.

Artículo aceptado:

24 de febrero de 2015.

\section{Resumen}

Se presenta una propuesta de indicadores ligados a la transparencia en las bibliotecas universitarias, identificando áreas informativas relevantes para los diversos colectivos o público interesado que tiene alguna relación con ellas (stakeholders). Se han tenido en cuenta las buenas prácticas identificadas en bibliotecas y otras organizaciones en esta materia. A continuación se analiza y valora la presencia de estos indicadores en la página web de las bibliotecas pertenecientes al consorcio madrileño Madroño para conocer su grado de transparencia. La comparativa entre el nivel de transparencia de algunas bibliotecas con el de sus respectivas universidades permite ver cierta relación entre

* Universidad Carlos III de Madrid, España.areyes@bib.uc3m.es

INVESTIGACIÓN BIBLIOTECOLÓGICA, Vol.30, Núm. 70, septiembre/diciembre, 2016, México, ISSN: 0187-358X. pp. 105-128 
ambas. Los resultados del análisis muestran que algunas bibliotecas pueden realizar mejoras significativas, fundamentalmente, en cuanto a visibilidad, actualidad de la información que ofrecen y, también, incorporando algunos contenidos relevantes para sus diferentes grupos de interés.

Palabras clave: Transparencia; Rendición de cuentas; Bibliotecas universitarias españolas; Indicadores; Consorcio Madroño.

\section{Abstract}

\section{Transparent universities with transparent libraries}

Ana R. Pacios

This paper proposes transparency indicators for university libraries, while identifying areas of information relevant to the diverse public stakeholders, in view of good practices identified in libraries and other organizations in this field. In order to determine the degree of transparency of these libraries belonging to the $\mathrm{Ma}$ droño consortium of Madrid, the existence of these indicators in their respective websites is assessed. A comparison of the degree of transparency of selected libraries to that of their respective universities reveals that some libraries could implement significant improvements, chiefly in the areas of visibility and currency of the information they offer, while also incorporating relevant contents for their diverse interest groups.

Keywords: Transparency; Accountability; Spanish University Libraries; Indicators; Madroño Consortium.

\section{INTRODUCCIÓN}

$\mathrm{D}$ esde el inicio de la crisis económica en el 2008, la transparencia, la rendición de cuentas de las organizaciones y el acceso libre a esta información están siendo objeto de una especial atención; se han convertido en un concepto "al alza" e incluso se consideran como un elemento clave de la 
cultura organizativa. ${ }^{1}$ Ciertas instituciones se están empeñando a fondo en el compromiso de impulsar la transparencia y la rendición de cuentas, por ejemplo, a nivel internacional, la coalición Transparency International; en España, las fundaciones Compromiso y Transparencia, Luis Vives, y Lealtad. Por otra parte, la reciente Ley de Transparencia española, ${ }^{2}$ cuya aplicación se inició en diciembre del 2014, muy controvertida y debatida por sus carencias y limitaciones, pretende garantizar el derecho de acceso a la información pública. Exige a los sujetos comprendidos en su ámbito de aplicación, entre ellos las universidades públicas, publicar información relativa a las funciones que desarrollan, la normativa que les resulta de la aplicación y su estructura organizativa, además de sus instrumentos de planificación y la evaluación de su grado de cumplimiento. En su capítulo II (dedicado a la publicidad activa) se insta a las administraciones públicas a publicar de forma periódica y actualizada la información que sea relevante para garantizar la transparencia de su actividad. En particular, debe estar a disposición la información de carácter institucional, organizativa y de planificación (art. 5), así como la de naturaleza económica, presupuestaria y estadística (art. 7).

Transparencia es un término que comprende la rendición de cuentas (accountability), la confianza y la credibilidad y su marco de actuación se sitúa en el de la evaluación, aunque también está ligada a otros conceptos como la responsabilidad social corporativa. La claridad de la información y el acceso libre a la misma son aspectos relacionados con todos ellos. La transparencia expresa la necesidad de que una organización cumpla con la responsabilidad de proporcionar información sobre la gestión y los resultados, justifique sus actuaciones y, en definitiva, rinda cuentas ante todas las personas que tienen una relación directa con ella o con sus grupos de interés (stakebolders). Es una práctica que forma parte del sistema democrático como resultado del derecho a saber en qué y cómo se utilizan los fondos públicos (eficacia y eficiencia).

Si una organización rinde cuentas voluntariamente genera confianza y credibilidad, y ello repercute de manera directa en su reputación de cara a la sociedad. Pero el hecho de rendir cuentas no debería verse como un fin en sí mismo sino como un medio para lograr una relación de confianza entre los que la dirigen y gestionan y los que participan activamente en ella, como son sus trabajadores, o los que se benefician de sus servicios o actuaciones. A pesar de esta necesidad manifiesta de rendir cuentas no se ha llegado a determinar la cantidad y calidad idónea de la información que deberían suministrar las organizaciones para hacerlo de modo conveniente.

1 J. Cabrera, "La transparencia es clave para el futuro de nuestras organizaciones".

2 Ministerio de la Presidencia, Ley 19/2013 de 9 de diciembre, de Transparencia, Acceso a la Información Pública y Buen Gobierno. 
Las TIC, y en particular la Web 2.0, constituyen una potente herramienta de comunicación y transparencia que permite compartir la información, además, de modo interactivo. La página web supone una identificación corporativa de la organización con todos los que tienen alguna relación con ella que sirve también como medio de publicidad. De ahí que pueda convertirse en el canal principal para rendir cuentas mostrando no sólo lo que hace, sino cómo lo hace y los resultados que consigue.

En el ámbito de las organizaciones no lucrativas, dedicadas a proporcionar servicios a la sociedad con el dinero público que reciben y que, en caso de generar algún beneficio, lo destinan al cumplimiento de su misión, la transparencia y la rendición de cuentas se asumen de un modo más amplio. Así lo revela el informe sobre la transparencia en organizaciones no lucrativas elaborado por el Observatorio del Tercer Sector (en adelante OTS) en el que se percibe como algo inherente a su razón de ser, vinculado a su misión y a sus valores. La rendición de cuentas supone para ellas, además de las connotaciones economicistas y las ligadas al cumplimiento de la legalidad, "dar cuentas a la sociedad del impacto que tienen sus actuaciones, sus actividades y de cómo ayudan a mejorar la sociedad, su compromiso con su misión (y por tanto, por qué hacen unas actividades y no otras)". 3

Estudios sucesivos sobre la transparencia de algunas instituciones españolas pretenden contribuir a incrementarla, identificando las áreas relevantes de información con especial trascendencia pública. La fundación Compromiso y Transparencia ha realizado dos exámenes consecutivos durante los años 2011 y 2012 en los que evalúa el grado de transparencia de 50 universidades públicas, añadiendo en el último el de 25 universidades privadas. ${ }^{4}$ En ellos se muestran las universidades más transparentes en forma de ranking atendiendo a determinados criterios y se recomienda a sus órganos de gobierno que presten más atención a la información institucional que se distribuye a través de la web, definiendo los contenidos relevantes desde el punto de vista de la rendición de cuentas a la sociedad. Teniendo en cuenta que las bibliotecas universitarias son uno de sus servicios básicos, además de uno de los centros de gasto de estas instituciones que gestionan "como mínimo el $5 \%$ del presupuesto total ordinario de la Universidad", 5 estaría justificado que se muestren transparentes. Es un aspecto que contribuye a afianzar

3 Observatorio del Tercer Sector, Transparencia y rendición de cuentas en las organizaciones no lucrativas, 13.

4 E. Barrio y J. Martin Cavanna, Examen de transparencia. Informe de transparencia en la web de las Universidades Públicas Españolas, y Examen de transparencia. Informe de transparencia en la web de las Universidades Públicas Españolas 2012. universitarias y científicas, 62-63. 
y aumentar su valoración y la buena reputación que tienen en la universidad. Partiendo de esta idea, surgen algunas preguntas que han llevado a realizar esta investigación:

- ¿Qué información debería proporcionar una biblioteca universitaria para mostrarse transparente?

- ¿Se muestran transparentes las bibliotecas universitarias a través de su página web?

- ¿Existe relación entre la filosofía de transparencia de la universidad y la de uno de sus servicios básicos como es la biblioteca?

\section{LA TRANSPARENCIA EN}

LAS BIBLIOTECAS UNIVERSITARIAS

La evaluación en las bibliotecas universitarias españolas es una operación afianzada en la mayoría, como lo demuestra la literatura profesional y el número de bibliotecas que han obtenido distintos reconocimientos a la calidad de sus servicios después de haberse sometido a procesos de evaluación. ${ }^{6}$ Por otra parte, en 1994 la Red de Bibliotecas Universitarias Españolas (REBIUN) acordó recoger datos relacionados con su actividad y desde entonces es posible consultar datos estadísticos e indicadores anuales, tanto predefinidos como a medida. Esto permite a cualquiera consultar y valorar aspectos relacionados con su rendimiento, eficacia, calidad e impacto. Este compromiso es, en principio, un ejercicio ligado a la transparencia de las bibliotecas universitarias españolas.

La literatura sobre la transparencia y la rendición de cuentas se relaciona con la de la evaluación, teniendo en cuenta que ambas forman parte de un mismo proceso. La rendición de cuentas y la evaluación son necesarias para que la calidad se reconozca y la financiación se justifique. ${ }^{7}$ Los antecedentes, etapas y avances en materia de evaluación de las bibliotecas han sido secuenciados por Heath, con énfasis en el apoyo que la American College and Research Libraries (ACRL) ha venido prestando a todo tipo de actividades relacionadas con este tema. ${ }^{8}$ Las Normas para Bibliotecas de Educación Superior, en el apartado relativo a la gestión y administración de la biblioteca,

6 Nuria Balagué, "Consolidando la calidad en las bibliotecas universitarias: evaluaciones, sellos, diplomas y certificaciones”.

7 J. M. Fluxá, "La rendición de cuentas y la evaluación son necesarias para que la calidad se reconozca y la financiación se justifique".

8 F. Heath, "Library assessment: the way we have grown". 
dicen textualmente: "The library communicates assessment results to library stakeholders". " Es una mención expresa a la rendición de cuentas ante cualquiera que tenga que ver con ellas. No obstante, aunque se evalúe y se rindan cuentas, en ocasiones falla la difusión: no se da, se da poco a conocer o no se hace del modo adecuado. A esto se une el hecho de que, al igual que sucede en el mundo empresarial, contamos con mucha información de las bibliotecas, datos e indicadores, pero faltan cuestiones críticas que permitan ver la estrategia y proyección de futuro que tienen.

Los profesionales de las bibliotecas se han referido a la necesidad de rendir cuentas ante la competencia con otros servicios de la universidad, así como la información que se debería proporcionar, caso de St. Clair, ${ }^{10}$ Osman y Bidin ${ }^{11}$ y Smith. ${ }^{12}$ Otros se han mostrado críticos con el uso que se hace de todos los datos que recogen las bibliotecas cuando sólo sirven para demostrar su valor y rendir cuentas ante el equipo de gobierno de la universidad, y no se diseñan y utilizan para la mejora de los servicios y el aprendizaje organizacional. $^{13}$

En España, el tema de la transparencia en las bibliotecas viene interesando, igualmente, desde hace tiempo. Carmena propuso en la década de los 90 justificar las decisiones que se tomaran en las bibliotecas mediante la elaboración de memorias-informes y proporcionó las pautas a seguir para redactarlos. ${ }^{14}$ También ha sido el tema de diferentes foros en los que se presentaron documentos, como las "cartas de servicios", para contribuir, entre otras cuestiones, a la transparencia administrativa en las bibliotecas. ${ }^{15}$ Con motivo de la crisis, se ha vuelto a hacer hincapié en la necesidad de que las bibliotecas rindan cuentas a la comunidad sobre el mejor y más eficaz uso de los recursos puestos a su disposición. ${ }^{16}$

Aunque no existe una normativa que determine qué tipo de documentos o información deberían mostrar las bibliotecas, algunos reglamentos hacen mención expresa a la obligación de elaborar un informe o memoria anual

9 ACRL (American College and Research Libraries), Standards for Libraries in Higher Education.

10 G. St. Clair, "Assessment in an Era of Accountability".

11 Z. Osman y S. Hanon Bidin, "Accountability in library management: issues and strategies for the 21st. Century".

12 Gregory A. Smith, Retooling the Profession: Librarianship in an Era of Accountability and Competition.

13 Meredith Farkas, "Accountability vs. Improvement: Seeking Balance in the Value of Academic Libraries Initiative".

14 Miguel A. Carmena Escribano, "Tomar decisiones en una biblioteca: algunas sugerencias sobre las posibles pautas a seguir en la elaboración de memorias-informes".

15 Julia García Maza, "Exigencia de transparencia vs. vocación de opacidad: función social de las cartas de servicios en las bibliotecas".

16 Cristóbal Pasadas Ureña, "Multialfabetización, aprendizaje a lo largo de la vida y bibliotecas". 
del servicio. ${ }^{17}$ Esto supone que la biblioteca universitaria, responsable de la gestión y ejecución de un presupuesto, rinda cuentas ante su institución de cómo lo ha gestionado; práctica habitual, por otra parte, entre las universidades.

REBIUN también ha puesto interés en la transparencia, como demuestra su III Plan Estratégico, que incluye un objetivo dedicado a la coordinación de las memorias de actuación, estadísticas e indicadores como modelo de transparencia y herramienta de gestión de la calidad.

\section{OBjetivos y MEtodología}

Este trabajo tiene dos objetivos. El primero pretende determinar la información que debería mostrar una biblioteca ligada a la transparencia, teniendo en cuenta las buenas prácticas identificadas en bibliotecas y en otras organizaciones en esta materia. Se presenta una propuesta de indicadores ligados a áreas relevantes así como ejemplos de su presencia en determinadas bibliotecas. Se entiende el indicador como la unidad de información (documento, dato o símbolo) que muestra o indica lo que se pretende en cada área a efectos de transparencia.

El segundo objetivo es, aplicando la propuesta realizada, conocer el nivel de transparencia que muestran las bibliotecas universitarias pertenecientes al consorcio Madroño a través de su página web. Para ello se analiza la presencia de los indicadores establecidos. Esto servirá, además, para ver si es posible vincular la transparencia que muestra la web de la universidad y la de su biblioteca. Se parte de la hipótesis de que la filosofía y cultura de transparencia de la primera debería influir y ser secundada por la última.

Para la realización de este trabajo se ha tomado como modelo el ya referido de Barrio y Cavanna ${ }^{18}$ sobre la transparencia en las universidades españolas. Siguiendo la metodología utilizada en sus dos ediciones, en primer lugar se comenzó eligiendo el grupo de bibliotecas objetivo del análisis. En segundo lugar, se identificaron las áreas más relevantes sobre las qué rendir cuentas de acuerdo con la misión y funciones de una biblioteca universitaria. A continuación se seleccionaron los contenidos o unidades básicas de información (indicadores) correspondientes a cada una de las áreas elegidas te-

17 Por ejemplo, el Reglamento del Servicio de Biblioteca de la Universidad Autónoma de Barcelona.

18 Barrio y Martin Cavanna, Examen de transparencia. Informe de transparencia en la web de las Universidades Públicas Españolas, y Examen de transparencia. Informe de transparencia en la web de las Universidades Públicas Españolas 2012. 
niendo en cuenta algunos ejemplos observados en bibliotecas universitarias tomadas como referente. Después de establecer estos indicadores se analiza su presencia en cada una de las páginas web de las siete bibliotecas universitarias pertenecientes, con pleno derecho, al consorcio Madroño, elegidas como muestra sobre la qué trabajar. Se tomó este grupo por tratarse de bibliotecas de universidades públicas madrileñas, diferentes en tamaño, número de usuarios, colección, infraestructuras, etc. ${ }^{19}$ La búsqueda de los indicadores se ciñó a su página web dado que es la herramienta principal de la biblioteca para acceder a sus recursos, además de a sus servicios y, por tanto, también para consultar este tipo de información relacionada con su gestión.

Para estimar idónea la información desplegada en las páginas web de las bibliotecas se han tenido en cuenta cuatro criterios habitualmente considerados en la evaluación de sedes web: ${ }^{20}$

- Visibilidad. Que la información se localice en un lugar fácilmente visible y los contenidos sean reconocibles; que no haya que recorrer un camino largo para llegar allí.

- Accesibilidad. Entendida como la posibilidad o facilidad de llegar a la sede web, además de los factores que facilitan su uso o disponibilidad. Se traduce en que pueda ser transformable, utilizable en cualquier dispositivo de navegación, comprensible, navegable y que se interactúe con ella.

- Actualidad. Que la información esté al día, verificable a través de la fecha de actualización de los documentos. La información requerida debía corresponder al año 2013 por ser el último cerrado legalmente cuando se elaboró el trabajo. Puesto que el análisis se realizó en mayo del 2014 y las memorias de las bibliotecas correspondientes a ese ejercicio aún no estaban publicadas se decidió considerar como actual el año 2012.

- Comprensible. La información debe ser entendible por aquellos que la consultan. Tiene, por tanto, que estar en castellano o darse la opción de traducir esa información en caso de que estuviera en otra lengua.

19 Después de ser aceptado este trabajo, la biblioteca de la Universidad Complutense de Madrid dejó de pertenecer al mencionado consorcio, hecho que no condicionó su presencia en la investigación ya realizada puesto que en el momento del análisis era un miembro de pleno derecho.

20 Marina Jiménez Piano, "Evaluación de sedes web”. 


\section{ÁREAS DE INFORMACIÓN RELEVANTE LIGADAS}

\section{A LA TRANSPARENCIA EN LAS BIBLIOTECAS}

Partiendo del estudio al que se ha hecho alusión, se presenta la relación de áreas de información consideradas relevantes con los indicadores que una biblioteca universitaria debería proporcionar para mostrarse transparente a través de su página web (Tabla I), así como algunos ejemplos representativos de cada uno. Se ha tenido en cuenta en esta adaptación su naturaleza peculiar, considerando su relevancia para los grupos de interés a los que puede interesar esa información: políticos, equipo de gobierno de la universidad, instituciones u organismos que contribuyen a su financiación, los beneficiarios de sus servicios (usuarios reales y potenciales, profesores y alumnos, especialmente), sus propios trabajadores, otras bibliotecas con las que coopera, proveedores o suministradores de recursos y público en general.

Tabla I. Indicadores de transparencia propuestos agrupados por áreas informativas relevantes

\begin{tabular}{|c|c|}
\hline Áreas informativas & Indicadores \\
\hline \multirow{2}{*}{$\begin{array}{l}\text { 1. Propósito del servicio y } \\
\text { objetivos que persigue }\end{array}$} & a) Definición de la misión \\
\hline & b) Plan estratégico \\
\hline \multirow{4}{*}{$\begin{array}{l}\text { 2. Órganos de gobierno y } \\
\text { reglas de funcionamiento }\end{array}$} & c) Miembros que integran la comisión de la biblioteca \\
\hline & d) Reglamento \\
\hline & e) Normativa específica de funcionamiento de los servicios \\
\hline & f) Carta de deberes y derechos de los usuarios \\
\hline 3. Oferta de servicios & g) Carta de servicios \\
\hline \multirow{2}{*}{$\begin{array}{l}\text { 4. Los recursos de } \\
\text { información }\end{array}$} & h) Política/programa de gestión de la colección \\
\hline & i) Política institucional de acceso abierto \\
\hline \multirow[t]{2}{*}{ 5. Personal } & j) Organigrama \\
\hline & k) Directorio de personal \\
\hline \multirow[t]{4}{*}{ 6. Resultados } & I) Indicadores de gestión (cuadro de mando) \\
\hline & m) Encuestas de satisfacción \\
\hline & n) Memoria anual o informe de actividades \\
\hline & o) Reconocimientos, premios, certificaciones \\
\hline \multirow[t]{2}{*}{ 7. Información económica } & p) Presupuesto \\
\hline & q) Contratos de licitación y concursos \\
\hline $\begin{array}{l}\text { 8. Pertenencia a redes y } \\
\text { otras relaciones de } \\
\text { colaboración }\end{array}$ & r) Redes con las que se trabaja y coopera \\
\hline
\end{tabular}

\section{Propósito del servicio de biblioteca y objetivos que persigue}

El propósito o la razón de ser del servicio se concreta en la misión, declaración que se define cuando se aborda un proceso de planificación estratégica, junto a la visión y los valores. Debe revisarse y, si es preciso, modificarse cada cierto tiempo para adaptarla a las circunstancias particulares del entorno. En cual- 
quier caso, tiene que estar alineada con los objetivos de la universidad y orientarse hacia el compromiso estratégico. ${ }^{21}$ Debería mostrarse en un lugar bien visible de la página web con las correspondientes fechas de adopción y revisión, ejemplo de la biblioteca del Instituto Tecnológico de Massachusetts (MIT). ${ }^{22}$

Contar con un plan estratégico demuestra que la biblioteca se orienta hacia objetivos, que involucra a las personas en un proyecto y que comunica lo que quiere hacer y cómo pretende hacerlo.

Los indicadores ligados a esta área informativa serían dos:

a) Definición de la misión.

b) Plan estratégico (en vigor).

\section{Órganos de gobierno y reglas de funcionamiento}

Esta área busca que se conozca el órgano encargado de elaborar las líneas de actuación de la política bibliotecaria, además de sus principales reglas de funcionamiento. En las universidades españolas la comisión de la biblioteca suele ser el órgano colegiado que participa en su planificación y gestión. Todos sus integrantes (presidente, secretario, representantes de docentes, del personal de administración y servicios, de los alumnos, etc.) deberían estar debidamente identificados por su participación en las decisiones que atañen a la biblioteca. Los ejemplos de Las Palmas ${ }^{23}$ y Jaén ${ }^{24}$ así lo hacen, esta última además permite el acceso a las actas de las reuniones celebradas.

En cuanto a las reglas que regulan el funcionamiento de la biblioteca, la fundamental es el reglamento, en el que se recogen las disposiciones relacionadas con la organización del servicio a las que deben atenerse tanto el personal de la biblioteca en sus funciones como los usuarios del servicio. También se consideran aquellas normativas específicas complementarias al reglamento que regulan el funcionamiento de algunos servicios como el préstamo de libros, uso de salas de trabajo, préstamo de computadoras portátiles, tablets, etc., documentos puestos a disposición a través de la página web de las bibliotecas y que, con frecuencia, se encuentran reunidos bajo un mismo enlace. Otro documento que formaría parte de esta área sería la carta de derechos y deberes de los usuarios, cuyo objetivo es que el usuario conozca de forma clara y comprensible cómo se rige su relación con la biblioteca.

21 W. Lougee, "The diffuse library revisited: aligning the library as strategic asset", 614.

22 MIT Libraries, fecha de consulta:12 de mayo de 2014, http://libraries.mit.edu/about/

23 Organización, fecha de consulta: 12 de mayo de 2014, http://biblioteca.ulpgc.es/conocenos_ organizacion\#organos_gobierno

24 Comisión de biblioteca, fecha de consulta: 12 de mayo de 2014, http://www10.ujaen.es/conocenos/servicios-unidades/biblio/sobreelservicio/comision 
Los indicadores asociados a esta parcela informativa serían:

c) Miembros que integran la comisión de la biblioteca (u órgano colegiado correspondiente).

d) Reglamento.

e) Normativa específica de funcionamiento de los servicios.

f) Carta de deberes y derechos de los usuarios.

\section{Oferta de servicios}

Si se habla de transparencia, además de mostrar la relación de servicios que presta a sus usuarios, la biblioteca debería "informar de los derechos que les asisten y de los compromisos que tiene relacionados con su prestación”, tal y como establece el Real Decreto 951/2005 del Ministerio de Administraciones Públicas de España, por el que se establece el marco general para la mejora de la calidad en la Administración General del Estado. En este sentido, el documento informativo más completo, y también el indicador ligado a esta información relativa a la prestación de los servicios, que refuerza los principios de transparencia y publicidad hacia los grupos de interés, es

g) La carta de servicios.

Las buenas prácticas en materia de evaluación de la calidad en las bibliotecas universitarias españolas muestran que son numerosas las que cuentan con él. De esta forma dan a conocer los servicios que prestan, los compromisos de calidad que adquieren en relación con ellos y también los sistemas o mecanismos que los usuarios pueden utilizar para comunicarse con la biblioteca (quejas y sugerencias, por ejemplo), además de las unidades de medida o indicadores para verificar el grado de cumplimiento de estos compromisos.

\section{Los recursos de información}

La colección de la biblioteca es un recurso imprescindible para la prestación de la mayoría de los servicios, además de suponer, generalmente, su inversión anual más importante. El desarrollo de las colecciones ha de ser un proceso transparente y las políticas en las que se base deben hacerse públicas. ${ }^{25} \mathrm{La}$ herramienta e indicador que ayuda a su correcta gestión y desarrollo y sirve

25 Ministerio de Cultura, Pautas para establecer una política de colecciones en una biblioteca pública. 
para la elaboración del presupuesto, distribuyéndolo con base en las necesidades reales y a los objetivos que se persiguen ligados a la colección, al usuario y a la institución, es

h) Política/programa de gestión de la colección.

Sirve para comunicar al usuario las prioridades y políticas relacionadas con todo lo que concierne a la colección. Muestra, entre otras cuestiones, los procedimientos relativos a la adquisición del material, a su integración en colecciones coherentes, al control de su crecimiento y mantenimiento, y al expurgo cuando proceda. ${ }^{26}$ Sobre su contenido se siguen haciendo propuestas para adaptarlas a los nuevos procedimientos actuales. ${ }^{27}$ Como indicador ligado a la transparencia, debería contener un apartado en el que se dé a conocer la fórmula elegida por la biblioteca para realizar el reparto del presupuesto entre los departamentos, facultades u otras unidades organizativas de la universidad según su estructura, así como los criterios establecidos que aplica.

Dado que la universidad es uno de los centros principales productores de conocimiento como resultado de la actividad investigadora, tendría que mostrar su compromiso con la difusión y el acceso abierto a este conocimiento a través de una política institucional. Pero es la biblioteca la que gestiona una buena parte de los materiales procedentes de la investigación generada en la universidad a través de los repositorios institucionales, ocupándose de su archivo y difusión. Por esta razón, otro indicador de transparencia ligado a esta área sería

i) La política institucional de acceso abierto a los resultados de la investigación.

\section{Personal}

La posibilidad de contactar con el personal que presta servicio en las administraciones públicas es un rasgo de acercamiento a la ciudadanía que sin duda contribuye a mejorar las relaciones entre ambos. Al igual que otras instituciones, muchas bibliotecas dan a conocer su estructura organizativa, las relaciones y dependencia entre las unidades y las funciones del personal, además de facilitar el contacto. Para ello ponen a disposición de los usua-

26 Aurora de Mesa Sánchez y Margarita Taladriz Más, "El concepto de colección y el programa para su gestión y desarrollo".

27 Holly Hibner, Making a collection count: a bolistic approach to library collection management, $20-35$. 
rios una serie de herramientas que podrían identificarse como indicadores de transparencia en este apartado:

j) Organigrama.

k) Directorio de personal.

Un buen ejemplo de organigrama es el de la biblioteca de Ohio, de tipo vertical con informaciones complementarias y relacionadas entre sí, además de varias páginas relacionadas con su estructura. ${ }^{28}$ Por lo que respecta al directorio, el de la biblioteca de la universidad de Harvard ofrece la posibilidad de búsqueda por áreas temáticas, bibliotecas, nombre del bibliotecario, lenguas y facultades o escuelas, ${ }^{29}$ además de mostrar una completa información de cada bibliotecario. Otro ejemplo modélico de presentación de sus bibliotecarios temáticos es el que hace la biblioteca del MIT bajo un enlace titulado Ask the expert. ${ }^{30}$

\section{Resultados}

Referirse a resultados supone aludir a evaluación y rendimiento. Cada cierto tiempo se presentan modificaciones y nuevas alternativas, además de propuestas en cuanto a métodos y técnicas para medir los resultados de las bibliotecas. ${ }^{31}$ Desde la International Standard Organisation (ISO) se ha tratado de promover el uso de indicadores de rendimiento y normalizar la recolección de información para su cálculo, a través de tres ediciones. ${ }^{32}$

Las bibliotecas universitarias españolas tienen definidos los datos estadísticos e indicadores anuales que recogen para evaluar su rendimiento, accesibles a través de la página web de REBIUN. ${ }^{33}$ En este trabajo interesa verificar si hacen uso de alguno de estos indicadores para el control de su gestión mediante un cuadro de mando y si esta información está disponible en su página web, dado que esta herramienta se ha vinculado en diferentes ocasiones

28 Organizational Chart Obio Library, fecha de consulta: 12 de mayo de 2014, http://www.docs toc.com/docs/49249554/The-Ohio-State-University-Libraries-Organizational-Chart; University Libraries Committee Structure, fecha de consulta: 12 de mayo de 2014, http://library.osu. edu/about/osul-committees/university-libraries-committee-structure/

29 Staff Library, fecha de consulta: 16 de mayo de 2014, http://lib.harvard.edu/staff-directory

30 Ask the Expert, fecha de consulta: 16 de mayo de 2014, http://libraries.mit.edu

31 P. Brophy, Measuring library performance: principles and techniques.

32 Véase el trabajo Nuevos instrumentos para la evaluación de bibliotecas: la normativa internacional ISO sobre la tercera y última ISO 11620:2014, coordinado por Marta de la Mano González.

33 Indicadores REBIUN, fecha de consulta: 16 de mayo de 2016, http://estadisticas.rebiun.org/ cuestionarios/indicadores/indicadores_main.asp\# 
con la transparencia en la gestión pública. ${ }^{34}$ Mediante este informe periódico, que prioriza la síntesis y la relevancia renunciando a la exhaustividad, se puede hacer un seguimiento de los objetivos que deberían alinearse con la estrategia de la organización, ${ }^{35}$ bien sea en su versión francesa (Tableau de Bord) o americana (Balanced Scorecard). Asociaciones como la ACRL ${ }^{36}$ han impulsado su uso y hay bibliotecas con una larga trayectoria en su utilización. ${ }^{37}$ En España, la Pompeu Fabra lo muestra en su página web desde finales de los años 90; ${ }^{38}$ también la Carlos III, que lo adoptó en el 2003 para el control y seguimiento de la gestión del servicio. ${ }^{39}$

Teniendo en cuenta la variada información relacionada con los resultados de una biblioteca los indicadores ligados a la misma se han centrado en tres aspectos:

1) Cuadro de mando.

m) Resultados de las encuestas de satisfacción a los usuarios.

n) La memoria anual o informe de actividades.

Al igual que otras organizaciones, las bibliotecas suelen elaborar una memoria anual en la que dan cuenta de las actividades realizadas a lo largo del año a modo de balance. Si es completa, puede contener información relacionada con todos los aspectos sobre su rendimiento: estadísticas sobre el uso de los servicios, indicadores, resultados de las encuestas de satisfacción de los usuarios, grado de consecución o cumplimiento de los objetivos del plan estratégico, proyectos de cooperación, las cifras anuales relacionadas con usuarios, horarios, instalaciones, etc., sin olvidar la información económico-financiera derivada del presupuesto aprobado y ejecutado por la biblioteca, mostrando las partidas de ingresos, gastos e inversiones y la asignación de recursos destinados a los distintos planes y proyectos. Toda esta información se presenta y estructura respondiendo a criterios diversos según la biblioteca en cuestión.

34 Por ejemplo, Carlos Vivas Urrieta, El Cuadro de Mando Integral en el sector público local: el caso del Ayuntamiento de San Cugat del Vallés.

35 José Bisbe y Viñas, "Criterios y herramientas para el control de la gestión a entidades no lucrativas", 148.

36 ACRL (American College and Research Libraries), Library Assessment Conference: Building Effective, Sustainable, Practical Assessment.

37 Balanced Scorecard, fecha de consulta: 20 de mayo de 2014, http://www2.lib.virginia.edu/ $\mathrm{bsc} / \mathrm{metrics} / \mathrm{index} \cdot \mathrm{html}$

38 Indicadors, fecha de consulta: 20 de mayo de 2014, http://www.upf.edu/bibtic/es/coneixer/ indicadors/

39 Teresa Malo de Molina, "El Cuadro de Mando Integral, una herramienta para visualizar la estrategia en la biblioteca”, 56. 
En esta área vinculada a los resultados hay que tener en cuenta también la información ligada a aspectos cualitativos que muestran "cómo hace las cosas la biblioteca”. Por ello, indicadores que evidencian la calidad de sus servicios, procesos y actividades, serían

o) Reconocimientos, premios, certificaciones obtenidos.

\section{Información económica}

Esta información es la que más se relaciona con la rendición de cuentas, de ahí la fuerte connotación económica que se vincula fundamentalmente con

p) El presupuesto que se gestiona, así como los datos de su ejecución.

Los responsables de las bibliotecas, como gestores de fondos públicos, demuestran profesionalidad al informar y justificar la actividad económico-financiera que han realizado durante el año dando a conocer el presupuesto asignado y ejecutado al finalizar dicho periodo, desglosado por capítulos y conceptos. Aunque es habitual que lo incluyan en la memoria anual, hay diferencias notables en cuanto al nivel de detalle que ofrecen, por ejemplo, en lo relativo al personal. Dado que suele ser un gasto que la biblioteca no gestiona, a excepción del sueldo percibido por los estudiantes becarios (incluido en el cap. IV), muchas no ofrecen información al respecto con lo cual si se pretende conocer este dato hay que recurrir a otras fuentes (la memoria de la universidad o la página de REBIUN de datos e indicadores). Bibliotecas como las de la Rioja ${ }^{40}$ y Las Palmas ${ }^{41}$ sí lo hacen.

Si la biblioteca cuenta con financiación alternativa a la procedente de universidad, sería oportuno informar del nombre de las empresas o instituciones colaboradoras, de las subvenciones y ayudas públicas concedidas con indicación de su importe; ejemplo de la biblioteca universitaria de Yale. ${ }^{42}$

Otros indicadores de transparencia en relación con esta área vinculada a la información económica serían:

q) Los contratos de licitación y concursos.

40 Memoria de la biblioteca 2013, fecha de consulta: 20 de mayo de 2004, http://biblioteca.unirioja.es/documentos/burmen13.pdf

41 Memoria informe 2012, fecha de consulta: 20 de mayo de 2014, https://biblioteca.ulpgc.es/ files/repositorio_de_docum152/memorias/bu/memoria_2012.pdf

42 Yale University Library. Annual Report 2012-13, fecha de consulta: 20 de mayo de 2014, http://elischolar.library.yale.edu/cgi/viewcontent.cgi?article=1006\&context=yul_annual-reports\#page $=2$ 
En España cualquier organismo o institución pública está obligado a sacar a concurso, y por tanto publicitar, los contratos con proveedores a partir de una determinada cantidad para cumplir con la legislación básica del Estado en materia de contratos públicos. También las bibliotecas tienen que convocar contrato público para la suscripción de revistas y otras publicaciones, cualquiera que sea su soporte, así como la contratación del acceso a la información contenida en bases de datos especializadas y otros recursos electrónicos cuando la cuantía supere los 200.000,00 €. Generalmente, las universidades disponen de una página web desde la que se accede al "perfil de contratante ${ }^{\text {,43 }}$ en la que se publican las adjudicaciones de los contratos, también los de las bibliotecas, así como otros datos e informaciones referentes a su actividad contractual, de acuerdo con lo establecido en la Ley de Contratos del Sector Público. La Ley de Transparencia también recoge este aspecto (art. 8, 1a) y especifica que esta información se debe dar a conocer indicando la duración, el importe, los licitadores participantes y la identidad del adjudicatario.

La biblioteca de la Universidad de Barcelona, por ejemplo, da a conocer en su página web los contratos y concursos relativos a la provisión de recursos de información, y muestra información sobre la convocatoria, objetivo, plazos y resolución. ${ }^{44}$ Por su parte, la British Library se puede considerar un buen ejemplo de transparencia al contar con una página, que se actualiza mensualmente, en la que publica los detalles de todos los pagos a proveedores superiores a $25.000 £ .{ }^{45}$

\section{Pertenencia a redes y otras relaciones de colaboración}

Trabajar en red y formar parte de organizaciones de segundo nivel tiene implicaciones de transparencia y rendición de cuentas ante aquellas con las que se colabora. Más aún, teniendo en cuenta que suele tener consecuencias económicas para las bibliotecas, por las cuotas que deben pagar por su pertenencia y así poder disfrutar de los beneficios que ésta conlleva. De ahí que otro indicador de transparencia sería mostrar e informar de

r) Las redes con las que se trabaja y coopera.

Es también una práctica habitual que siguen las bibliotecas universitarias españolas al mostrar en su página web el logo de las redes de las que forman

43 Perfil del contratante, fecha de consulta: 20 de mayo de 2014, http://www.uc3m.es/portal/ page/portal/perfil_contratante

44 Licitacions $i$ concursos, fecha de consulta: 14 de mayo de 2014, http://crai.ub.edu/ca/coneixel-crai/crai-unitats/unitat-administracio/licitacions-concursos

45 Transparency: Financial Transactions over £25,000, 14 de mayo de 2014, http://www.bl.uk/ aboutus/foi/transparency/transactionsover $25 \mathrm{k} /$ transactionsindex.html 
parte y con las que cooperan, así como el de otras instituciones con las que tienen acuerdos de colaboración, aportando información relacionada con su actividad (objetivos, servicios, etc.).

\section{LA TRANSPARENCIA EN LAS BIBLIOTECAS UNIVERSITARIAS DEL CONSORCio Madroño a TRAVÉS DE SU PÁGINA WEB}

La información ligada a la transparencia en las bibliotecas que pertenecen al consorcio Madroño se localiza, con mayor o menor visibilidad, agrupada bajo enlaces de diversa denominación (Sobre nosotros, Normativa y documentos, Organización y gestión, La biblioteca, Conocer la biblioteca, Conócenos), estructurada, a su vez, en distintos niveles. En un único caso, el de la UNED, se encontró un enlace en su página principal titulado Transparencia cuyos contenidos responden a esta intención.

Basándose en las áreas informativas establecidas y los 18 indicadores asociados a las mismas, su análisis en las siete bibliotecas muestra la situación que aparece en la Tabla II, cuyos aspectos más destacados se comentan a continuación:

- Los valores obtenidos en cada biblioteca oscilan en una escala que se sitúa entre 7 y 13 puntos (sobre un total de 18), lo que muestra diferencias significativas entre ellas. Las más transparentes son la de Alcalá, Carlos III y UNED, estas últimas con la misma puntuación.

- Los indicadores comunes a todas las bibliotecas son la normativa de uso de los servicios, el directorio de personal y la misión, a pesar de que en esta última falla su visibilidad al localizarse, salvo excepciones, en un segundo o tercer nivel dentro de otros documentos, además de no presentar fechas relacionadas con su adopción y actualización. En algún caso, no responde a los requisitos técnicos requeridos para formular esta declaración.

- Los indicadores ausentes en la muestra son el cuadro de mando y los contratos de licitación y concursos. Aunque el uso de los indicadores de gestión está generalizado en todas, en ningún caso están vinculados a una herramienta de pilotaje de toda la biblioteca basada en la "síntesis" como requiere un cuadro de mando. En el caso de la universidad Carlos III, que sí lo ha venido utilizando con este fin, ${ }^{46}$ no está el correspondiente al año objeto de este análisis. 


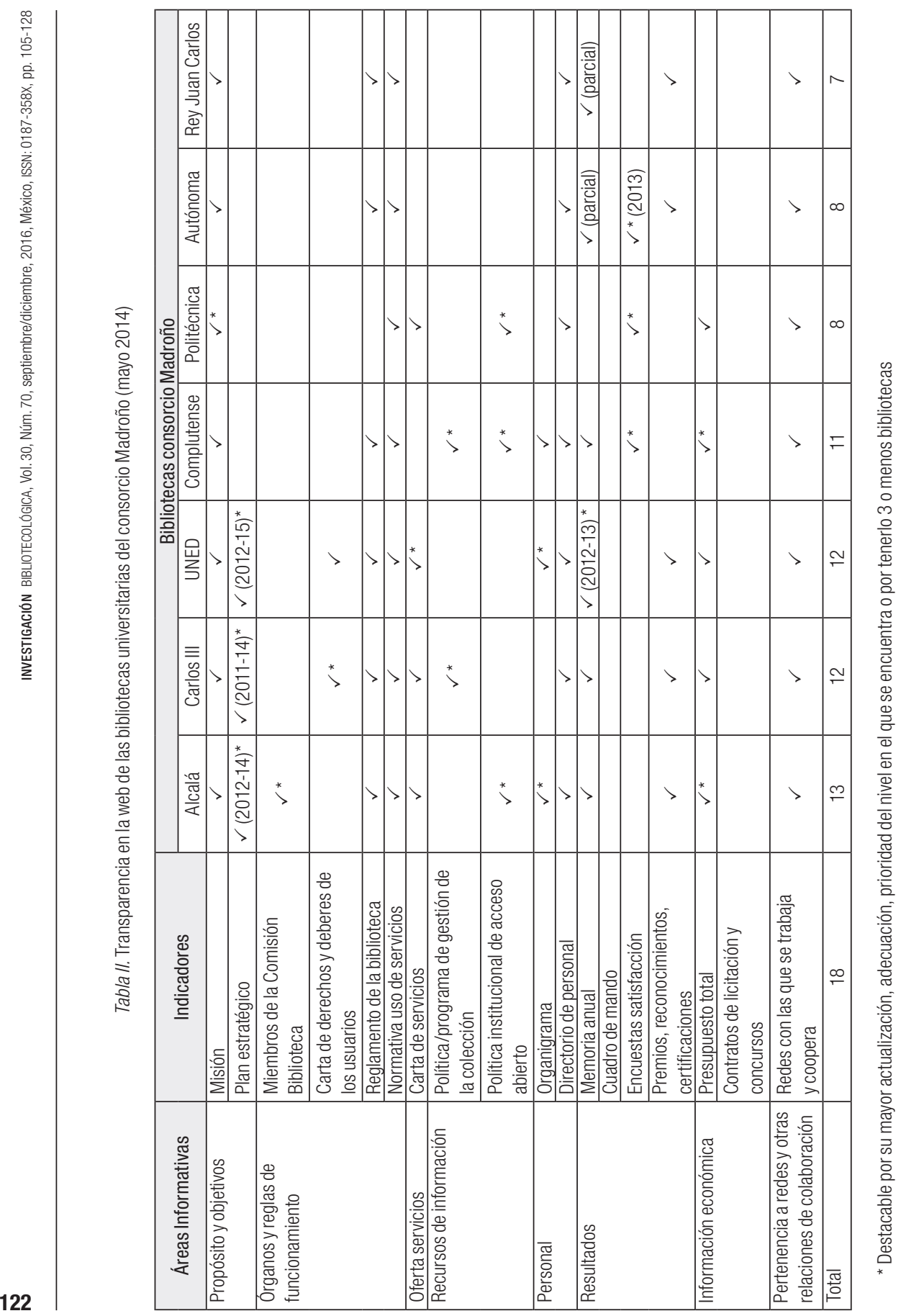


- Hay indicadores presentes en un reducido número de bibliotecas: los miembros de la comisión de la biblioteca sólo en la de Alcalá y con acceso a las actas; la política/programa de gestión de la colección (Carlos III y Complutense) y la carta de derechos y deberes de los usuarios (Carlos III y UNED); y la política institucional de acceso abierto, los planes estratégicos en vigor, el organigrama (vertical sólo en la UNED y Alcalá, este último más completo al incorporar nombres y niveles) y los resultados de las encuestas de satisfacción, en tres bibliotecas.

\section{CORRESPONDENCIA ENTRE LA TRANSPARENCIA}

\section{DE LAS UNIVERSIDADES Y LA DE SUS RESPECTIVAS BIBLIOTECAS}

En el último ranking de transparencia de las universidades españolas que se ha tomado como referente para este estudio, las pertenecientes al consorcio Madroño tienen un valor asignado en función de la presencia en la página web de la universidad de los 25 indicadores predefinidos en el trabajo. Todas las universidades se sitúan en un rango de valores que va desde el 24 al 3. Las madrileñas se sitúan en el intervalo del 21 al 11, como puede verse en las columnas de la izquierda de la Figura 1. Con el fin de observar un posible paralelismo entre la transparencia de las universidades y la de sus respectivas bibliotecas, teniendo en cuenta los 18 indicadores establecidos aquí para estas últimas, se asignó también un valor 1 a cada uno si estaba presente en su página web. Así se obtienen los valores que muestran las columnas de la derecha de la Figura 1, en un intervalo entre 13 y 7. A efectos de utilizar el ranking de universidades para establecer comparaciones no se ha realizado ponderación alguna en la valoración de la transparencia en las bibliotecas. Si se ponderara determinada casuística (por ejemplo, cuando la presencia de determinados indicadores se dé en tres bibliotecas o menos, caso de la política de gestión de la colección, el plan estratégico o la política institucional de acceso abierto) la situación de las que ocupan los primeros lugares se vería reforzada. 


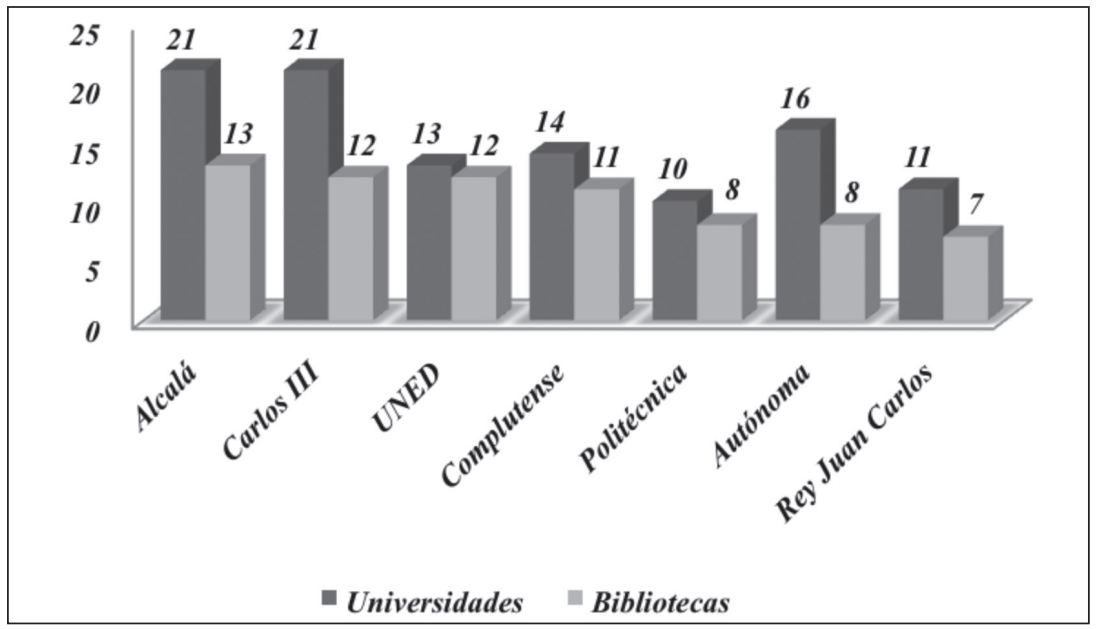

Figura 1. Comparativa, ranking de transparencia universidades-bibliotecas

Comparando los valores y el lugar que ocupan las universidades y sus respectivas bibliotecas se puede observar lo siguiente:

- Las dos bibliotecas mejor posicionadas (Alcalá y Carlos III) pertenecen a las dos universidades madrileñas más transparentes del ranking.

- En el resto se puede establecer cierta afinidad entre las universidades y sus bibliotecas aunque una se desvía claramente de esta tendencia. La universidad que ocupa el segundo puesto en transparencia (Autónoma) no tiene correspondencia por el puesto que ocupa su biblioteca. Pero la biblioteca que ocupa el último puesto pertenece a una de las universidades que ocupa uno de los lugares más bajos del ranking.

- Se podría afirmar que la cultura de transparencia de la universidad tiene un reflejo en la de su biblioteca, a pesar de que en un caso no resultaría válida esta afirmación.

\section{Conclusiones}

La presencia de los indicadores establecidos ligados a la transparencia en las páginas web de este grupo de bibliotecas universitarias madrileñas permite extraer una serie de conclusiones:

- Todas publican información que justifica su gestión y permite conocer cómo se organizan; sin embargo, hay diferencias relevantes entre ellas 
respecto a la visibilidad, actualización y puesta a disposición de terminados contenidos. Todas pueden mejorar en los tres criterios.

- La dispersión de los documentos asociados a los indicadores establecidos en muchos enlaces y niveles no facilita la visibilidad en ciertos casos; en otros, el tamaño de la letra es muy pequeño y su contraste débil, lo que unido a la saturación de contenidos repercute negativamente en la accesibilidad.

- La actualidad de la información falla en algunas, en particular con los planes estratégicos ya ejecutados sin continuidad en el momento actual, así como en las memorias o informes anuales que no se mantienen al día.

- Los contenidos mejor representados tienen que ver con la normativa de uso de los servicios, así como con la información relativa a su localización y contacto con los profesionales que allí trabajan.

- Las áreas que requieren una mejora atañen a los recursos de información. Tanto la política o programa de gestión de la colección como la relativa al acceso abierto tienen una escasa representación en la muestra.

- Los indicadores ligados a los resultados deberían mejorar en algunas bibliotecas. El presupuesto tiene que mostrar detalle de las partidas para que sea comprensible y fiable. Es importante, para conocer el costo total del servicio, que se incluya el capítulo relativo al gasto de personal.

- Aunque algunas presentan secciones útiles porque resumen de forma ordenada los datos más significativos de la biblioteca (presupuesto, visitantes, usuarios, colecciones, etc.) algunos necesitan de una explicación. No siempre los datos estadísticos o indicadores resultan comprensibles. En este sentido, la memoria o informe anual contribuye a aclarar convenientemente todos los datos añadiendo valor a las cifras.

- Un punto fuerte a destacar es el presupuesto dedicado a los recursos de información, muy detallado y bien diferenciado por tipología: monografías, revistas, recursos electrónicos (estos últimos, a veces según la modalidad de contratación), etc.

- A pesar de que las bibliotecas universitarias han consensuado indicadores que permiten llevar a cabo comparaciones, disponibles en REBIUN, ello no es óbice para que cada biblioteca tenga y muestre el grupo de indicadores relevantes ligados a su estrategia. En este sentido el uso de un cuadro de mando, además de ayudar al control de la gestión, permite ver las áreas clave de resultados de la biblioteca.

- Se puede inferir una relación entre la cultura de transparencia de la universidad y la de su biblioteca si se tiene en cuenta la correspondencia entre las mejor posicionadas en ambos casos. 


\section{REFERENCIAS BIBLIOGRÁFICAS}

ACRL (American College and Research Libraries). Library Assessment Conference: Building Effective, Sustainable, Practical Assessment, Baltimore, Maryland, October 25-27, 2010. Fecha de consulta: 12 de enero de 2014, http://libraryassessment.org/archive/2010.shtml

- Standards for Libraries in Higher Education, 2011. Fecha de consulta: 13 de febrero de 2014, http://www.ala.org/acrl/standards/ standardslibraries

Balagué, Nuria. "Consolidando la calidad en las bibliotecas universitarias: evaluaciones, sellos, diplomas y certificaciones”, El Profesional de la Información 16 (4) (2007): 338-342.

Barrio, E. y J. Martin Cavanna. Examen de transparencia. Informe de transparencia en la web de las Universidades Públicas Españolas, Madrid: Fundación Compromiso y Transparencia, 2012. Fecha de consulta: 10 de enero de 2014, http://www.compromisoempresa rial.com/wp-content/uploads/Universidades2012.pdf

- y J. Martin Cavanna. Examen de transparencia. Informe de transparencia en la web de las Universidades Públicas Españolas 2012, Madrid: Fundación Compromiso y Transparencia, 2013. Fecha de consulta: 10 de enero de 2014, http://www.compromisoytranspa rencia.com/upload/32/56/InformeUniversidad2012.pdf

Bisbe y Viñas, J. (1993), "Criterios y herramientas para el control de la gestión a entidades no lucrativas", Bibliotecas y servicios culturales en el ámbito municipal (Alcúdia, 18-20 de noviembre de 1992), 133-154. Alcúdia (Mallorca): Fundació Biblioteca d'Alcúdia.

Brophy, P. Measuring library performance: principles and techniques, London: Facet, 2006.

Cabrera, J. "La transparencia es clave para el futuro de nuestras organizaciones", Innovación en la gestión, 2008. Fecha de consulta: 10 de enero de 2014, http://blog.cabreramc.com/2008/10/09/la-trans parencia-es-clave-para-el-futuro-de-nuestras-organizaciones/

Carmena Escribano, Miguel A. "Tomar decisiones en una biblioteca: algunas sugerencias sobre las posibles pautas a seguir en la elaboración de memorias-informes", Boletín de la Asociación Andaluza de Bibliotecarios 33 (1993): 25-40.

Farkas, Meredith "Accountability vs. Improvement: Seeking Balance in the Value of Academic Libraries Initiative", OLA Quarterly, Measuring Succes 19 (1) (2013): 4-7.

Fluxá, J. M. "La rendición de cuentas y la evaluación son necesarias para que la calidad se reconozca y la financiación se justifique", $L a$ cuestión universitaria. Boletín electrónico de la cátedra UNESCO de gestión y política universitaria 3 (2007). Fecha de consulta: 19 de enero de 2014, http://www.lacuestionuniversitaria.upm.es/web/ grafica/videos/j_fluxa.pdf 
García Maza, Julia. "Exigencia de transparencia vs. vocación de opacidad: función social de las cartas de servicios en las bibliotecas", 109-118, en Actas 8 Jornadas españolas de documentación, FESABID, 2003.

Heath, F. "Library assessment: the way we have grown", en Library Quarterly 81 (1) (2011): 7-25.

Hibner, Holly. Making a collection count: a bolistic approach to library collection management. Oxford: Chandos, 2010.

Jiménez Piano, Marina. "Evaluación de sedes web", Revista Española de Documentación Científica 24 (4) (2001): 405-432. Fecha de consulta: 11 de enero de 2014, http://redc.revistas.csic.es/index.php/ redc/article/view/69/130

Lougee, W. "The diffuse library revisited: aligning the library as strategic asset”, Library Hi Tech 27 (4) (2009): 610-623.

Malo de Molina, Teresa. "El Cuadro de Mando Integral, una herramienta para visualizar la estrategia en la biblioteca", XII Jornadas Españolas de Documentación FESABID'13 (24-25 de mayo de 2013), 2013. Fecha de consulta: 23 de febrero de 2014, http://fesabid.org/ documentos/fesabid13-actas.pdf

Mano González, Marta. de la (coord.), Nuevos instrumentos para la evaluación de bibliotecas: la normativa internacional ISO, Madrid: aenor, 2014.

Mesa Sánchez, Aurora de y Margarita Taladriz Más. "El concepto de colección y el programa para su gestión y desarrollo", De libros y bibliotecas. Homenaje a Rocío Caracuel, 233-241. Sevilla: Universidad, 1995.

Ministerio de la Presidencia. Ley 19/2013 de 9 de diciembre, de Transparencia, Acceso a la Información Pública y Buen Gobierno (BOE, 10 de diciembre de 2013), 2013. Fecha de consulta: 11 de enero de 2014, https://www.boe.es/boe/dias/2013/12/10/pdfs/BOE-A-20 13-12887.pdf

Ministerio de Cultura. Pautas para establecer una política de colecciones en una biblioteca pública, 2009. Fecha de consulta: 22 de enero de 2014, http:/www.mcu.es/bibliotecas/docs/MC/ConsejoCb/ GruposTrabajo/GT_Seleccion_BP/09_Pautas_para_establecer_una _politica_de_colecciones_en_una_biblioteca_publica.pdf

OTS (Observatorio del Tercer Sector). Transparencia y rendición de cuentas en las organizaciones no lucrativas, Barcelona: OTS, 2007. Fecha de consulta: 23 de enero de 2014, http://www.lasociedadcivil.org/docs/ciberteca/200710_transparencia_ots_v44_copy.pdf

Osman, Z. y S. Hanon Bidin. "Accountability in library management: issues and strategies for the 21 st. Century", en Internacional Seminar Challenges of Libraries in the 21 st. Century (29-31 March. Manila, Philippines), 2004. Fecha de consulta: 13 de enero de 2004, http://asiapacific-odl2.oum.edu.my/C33/F219.pdf 
Pasadas Ureña, Cristóbal. "Multialfabetización, aprendizaje a lo largo de la vida y bibliotecas", Boletín de la Asociación Andaluza de Bibliotecarios, 98-99 (2010): 11-38.

REBIUN (Red Española de Bibliotecas Universitarias). Normas y directrices para bibliotecas universitarias y científicas, Madrid: Ministerio de Cultura, 1999. Fecha de consulta: 23 de enero de 2014, http:// bib.us.es/sobre_la_biblioteca/carta_servicios/normativa/common/ bibliotecas_universitarias_rebiun.pdf

Smith, Gregory A. Retooling the Profession: Librarianship in an Era of Accountability and Competition, Faculty Publications and Presentations, Paper 47, 2009. Fecha de consulta: 14 de febrero de 2014, http://digitalcommons.liberty.edu/lib_fac_pubs/47

St. Clair, G. "Assessment in an Era of Accountability", Journal of Academic Librarianship 24 (3) (1998): 197-198.

UAB (Universidad Autónoma de Barcelona). Reglamento del Servicio de Biblioteca, 2003. Fecha de consulta: 24 de enero de 2014, http:// www.uab.cat/servlet/Satellite/bibliotecas/normativas/reglamento -del-servicio-de-bibliotecas-1096482171114.html

Vivas Urrieta, Carlos. El Cuadro de Mando Integral en el sector público local: el caso del Ayuntamiento de San Cugat del Vallés, Madrid: Instituto Nacional de Administración Pública, 26 de junio de 2007. Fecha de consulta: 28 de junio de 2014, http://www.sfo. santcugat.cat/files/3-159454-annex/Doc02.pdf

Para citar este artículo:

Pacios, Ana R. 2016. "Universidades transparentes con bibliotecas transparentes." Investigación Bibliotecológica: Archivonomía, Bibliotecología e Información 70: 105-128. http://dx.doi.org/10.1016/ j.ibbai.2016.10.006 